\title{
105. A Unique Continuation Theorem of a Parabolic Differential Equation
}

\author{
By Taira SHIRota
}

Osaka University

(Comm. by K. KUnUGI, M.J.A., Oct. 12, 1959)

1. Introduction. Let $G$ be a convex domain of the euclidean $n+1$-space $R_{t, x}\left(-\infty<t<+\infty,-\infty<x_{i}<+\infty(i=1,2, \cdots, n)\right)$, containing a curve $C:\left\{\left(t, x_{i}(t)\right) \mid t \in[a, b]\right\}$, where $x_{i}(t) \in C^{2}[a, b]$.

Consider real solutions $u$ of an inequality of the following kind:

$$
\left|\frac{\partial u(t, x)}{\partial t}-a_{i j}(t, x) \frac{\partial^{2} u(t, x)}{\partial x_{i} \partial x_{j}}\right| \leqq M\left\{\sum_{1}^{n}\left|\frac{\partial u(t, x)}{\partial x_{i}}\right|+|u(t, x)|\right\} .
$$

Here $\left(\left(a_{i j}(t, x)\right)\right)$ denotes a positive definite, symmetric matrix of real valued functions $a_{i j}(t, x) \in C^{2}(G)$, and $M$ a constant.

Our purpose in this note is to prove the following theorem for solutions of (1.1).

Theorem. If $u$ is a solution of (1.1) in the convex domain $G$ and if for any $\alpha>0$,

$$
\lim _{\substack{r \rightarrow 0 \\ \max _{\mid x-x(t)=r}(t \in[-b, b]}}\left\{|u(t, x)|,\left|\frac{\partial u}{\partial t}(t, x)\right|,\left|\frac{\partial u}{\partial x_{i}}(t, x)\right|,\left|\frac{\partial^{2} u}{\partial x_{i} \partial x_{j}}\right|\right\}|x-x(t)|^{-\alpha}=0
$$

then $u$ vanishes identically in the horizontal component.

The method is based upon the ideas of H. O. Cordes [2] and E. Heinz [3]. The tools used are all elementary, but our proof is somewhat complicated.

2. The Cordes' transformation. Assuming $[a, b] \supset[-\varepsilon, 1+\varepsilon]$ $(\varepsilon>0)$, let $\AA(t)$ be the positive square root of the matrix $A(t)=$ $\left(\left(a_{i j}(t, x(t))\right)\right)$. Let

$$
x-x(t)=\AA(t) \widetilde{x} \quad \text { for } \quad t \in[-\varepsilon, 1+\varepsilon],
$$

then we may assume that for some $R_{1}>0$,

a) $a_{i k}(t, \widetilde{x}) \in C^{2}\left([-\varepsilon, 1+\varepsilon] \times D_{R_{1}}\right) \quad\left(D_{R_{1}}=\left\{x|| x \mid \leqq R_{1}\right\}\right)$,

b) $a_{i k}(t, 0)=\delta_{i k}$,

c) there are positive numbers $C_{1}$ and $C_{2}$ such that for any real vector $\left(\xi_{1}, \xi_{2}, \cdots, \xi_{n}\right)$

$$
C_{1} \sum_{1}^{n} \xi_{i}^{2} \leqq \sum a_{i j}(t, \widetilde{x}) \xi_{i} \xi_{j} \leqq C_{2} \sum_{1}^{n} \xi_{i}^{2}
$$

From (a), (b) and (c) we see the following

Lemma 1. For some $R_{2}, \widetilde{R}_{2}<R_{1}$ there is a topological transtormation from $[-\varepsilon, 1+\varepsilon] \times D_{R_{2}}$ onto $[-\varepsilon, 1+\varepsilon] \times D_{\hat{R}_{2}}$ :

$$
\tilde{y}=\tilde{y}(t, \widetilde{x}), t=t
$$

such that it satisfies the following conditions: 
I. 1) $\widetilde{y}(t, 0) \equiv 0$,

2) $\frac{\partial \widetilde{y}_{i}}{\partial \widetilde{x}_{j}}, \frac{\partial \widetilde{x}_{i}}{\partial \widetilde{y}_{j}}, \frac{\partial^{2} \widetilde{y}_{i}}{\partial \widetilde{y}_{j} \partial \widetilde{x}_{k}}, \frac{\partial^{2} \widetilde{x}_{i}}{\partial \widetilde{y}_{j} \partial \widetilde{y}_{k}}$ are continuous over $[-\varepsilon, 1+\varepsilon] \times$ $\left(D_{\widetilde{R}_{2}}-\{0\}\right)$ and

$$
\left|\frac{\partial \widetilde{y}_{i}}{\partial \widetilde{x}_{j}}\right|<C,\left|\frac{\partial \widetilde{x}_{i}}{\partial \widetilde{y}_{j}}\right|<C,\left|\frac{\partial^{2} \widetilde{y}_{i}}{\partial \widetilde{x}_{j} \partial \widetilde{x}_{k}}\right|<C|y|^{-1},\left|\frac{\partial^{2} \widetilde{x}_{i}}{\partial \widetilde{y}_{j} \partial \widetilde{y}_{k}}\right|<C|y|^{-1},
$$

3) $\frac{\partial \widetilde{y}_{i}}{\partial t}$ is continuous over $[-\varepsilon, 1+\varepsilon] \times\left(D_{\widetilde{R}_{2}}-\{0\}\right)$ and $\left|\frac{\partial \widetilde{y}_{i}}{\partial t}\right|<C$,

II. for any $\tilde{y}: 0<|\tilde{y}| \leqq \widetilde{R}_{2}$, there is a suitable polar coordinates $\left(r, \varphi_{0}\right)$ such that

$$
\frac{\partial}{\partial \widetilde{x}_{i}} a_{i j}(t, \widetilde{x}) \frac{\partial u}{\partial \widetilde{x}_{j}}=p(t, \widetilde{y})\left(\frac{\partial^{2}}{\partial r^{2}}+\frac{n-1}{r} \frac{\partial}{\partial r}+\frac{N}{r^{2}}\right) u+p_{i}(t, \widetilde{y}) \frac{\partial u}{\partial \widetilde{y}_{i}},
$$

where $p(t, \tilde{y}), p_{i}(t, \tilde{y})$ and the operator $N$ satisfy the following conditions:

1. $C>p(t, \tilde{y})>C^{-1},\left|p_{i}(t, \tilde{y})\right|<C$,

2. $|p(t, \widetilde{y})|<C,\left|\frac{\partial p(t, \widetilde{y})}{\partial t}\right|<C,\left|\frac{\partial p(t, \widetilde{y})}{\partial r}\right|<C,\left|\frac{\partial p(t, \widetilde{y})}{\partial \varphi_{\sigma}}\right|<C$,

3. $N=\frac{1}{\lambda(\widetilde{y})} \frac{\partial}{\partial \varphi_{\sigma}} \lambda(\widetilde{y}) \bar{a}_{\sigma \tau}(t, \tilde{y}) \frac{\partial}{\partial \varphi_{\tau}}, \lambda(y)=\frac{d O_{1}}{d \varphi_{1} d \varphi_{2} \cdots d \varphi_{n-1}}$,

where $d O_{1}$ is the usual surface element of the unit sphere,

4. there are two positive numbers $\bar{C}_{1}$ and $\bar{C}_{2}$ such that

$$
\bar{C}_{1} \sum_{1}^{n-1} \eta_{\sigma}^{2} \leqq \sum \bar{a}_{\sigma \tau}(t, \widetilde{y}) \eta_{\sigma} \eta_{\tau}=\bar{C}_{2} \sum_{1}^{n-1} \eta_{\sigma}^{2}
$$

for any real vector $\left\{\eta_{1} \cdots \eta_{n-1}\right\}$,

5. $\bar{a}_{\sigma \tau}, \frac{\partial \bar{a}_{o \tau}}{\partial t}, \frac{\partial \bar{a}_{o \tau}}{\partial r}$ and $\frac{\partial \bar{a}_{o \tau}}{\partial \varphi_{\rho}}$ are continuous and

$$
\left|\bar{a}_{\sigma \tau}\right|<C,\left|\frac{\partial \bar{a}_{o \tau}}{\partial t}\right|<C,\left|\frac{\partial \bar{a}_{\sigma \tau}}{\partial r}\right|<C,\left|\frac{\partial \bar{a}_{\sigma \tau}}{\partial \varphi_{\rho}}\right|<C,
$$

where the constants $\overline{C_{1}}, \bar{C}_{2}$ and $C$ depend only on $R_{1}, C_{1}, C_{2}$ and the derivatives of $a_{i j}(t, x)$ of order $\leqq 2$. (Here we use a finite number of fixed, suitable systems of polar-coordinates covering the unit sphere.)

To prove the above proposition, we only remark that

$$
\nu_{\sigma}\left(t, r, \theta_{1}, \theta_{2}, \cdots, \theta_{n-1}\right)=\frac{\sum a_{i k}(t, \widetilde{x}) \frac{\widetilde{x}_{i}}{r} \cdot \theta_{\sigma \mid \tilde{x}_{k}}}{\sum a_{i k}(t, \widetilde{x}) \frac{\widetilde{x}_{i}}{r} \cdot \frac{\widetilde{x}_{k}}{r}}
$$

satisfies the following conditions: for any $t \in[-\varepsilon, 1+\varepsilon]$ and $\widetilde{x}: 0 \leqq|\widetilde{x}|$ $<R_{1}$ the function $\nu_{\sigma}(t, r, \theta), \nu_{\sigma \mid r}, \nu_{\sigma \mid \theta_{\tau}}, \nu_{\sigma \mid t}, \nu_{\sigma \mid \theta_{\tau} \theta \rho}, \nu_{\sigma \mid r, \theta_{\tau}}$ and $\nu_{\sigma \mid t, \theta_{\tau}}$ are all continuous, and for any $t \in[-\varepsilon, 1+\varepsilon]$ and $\widetilde{x}: 0<|\widetilde{x}| \leqq R_{1}, \nu_{\sigma \mid r r}, \nu_{\sigma \mid r t}$ and $\nu_{\sigma \mid t t}$ are continuous, where $\nu(t, r, \theta)$ is considered as a function of $t, r, \theta$. Here and in the proof of the following sections $\left.u\right|_{h}$ denotes $\frac{\partial u}{\partial h}$. 
Furthermore by the transformation: $(t, \widetilde{y})=\left(t, r, \varphi_{\sigma}\right) \rightarrow\left(t, s, \varphi_{\sigma}\right)$ $=(t, y)$ :

we see the following

$$
s(r)=r e^{\int_{0}^{\left.r_{\left(e-m_{0} \tau\right.}-1\right) \frac{d \tau}{\tau}}}
$$

Lemma 2. By the transformation $(t, \tilde{y}) \rightarrow(t, y)$ with a sufficiently large $m_{0}$, the following condition is satisfied: for any $w \in C^{2}(y:|y|=1)$ and for any $t \in[-\varepsilon, 1+\varepsilon]$,

III. $\frac{\partial}{\partial s} \int N \omega \cdot \omega d O_{1} \leqq m_{0} \int N \omega \cdot \omega d O_{1}<0$

as well as Conditions I and II.

3. The first inequality. Using the above lemmas, we will deduce the Heinz' inequality with respect to (1.1). For this purpose we may assume that

$$
\begin{aligned}
L_{1}(u) & =q(t, x) \frac{\partial u}{\partial t}-a_{i \jmath}(t, x) \frac{\partial^{2} u}{\partial x_{i} \partial x_{j}}+b_{i}(t, x) \frac{\partial u}{\partial x_{i}} \\
& =q(t, x) \frac{\partial u}{\partial t}-\left(\frac{\partial^{2}}{\partial r^{2}}+\frac{n-1}{r} \frac{\partial}{\partial r}+\frac{1}{r^{2}} N\right) u,
\end{aligned}
$$

where $q(t, x)(>\delta>0) \in \bar{C}^{1}\left(t, r, \varphi_{o}\right), \quad a_{i j}(t, x) \in \bar{C}^{0}(t, x), \quad b_{i}(t, x) \in \bar{C}^{0}(t, x)$ and the coefficients of $N \in \bar{C}^{1}\left(t, r, \varphi_{\sigma}\right) \quad(0<r \leqq R)$ for fixed, suitable polar coordinates $\left(r, \varphi_{\sigma}\right)$ of $x$.

Furthermore we may assume that $u$ satisfies the condition (1.2) with $x_{i}(t)=0$ for $t \in[-\varepsilon, 1+\varepsilon]$.

Put $D_{r_{0}, K_{0}}=\left\{(t, x) \mid 0 \leqq t \leqq 1\right.$ and $\left.|x| \leqq r_{0} \wedge K_{0}{ }^{-1} t\right\}$ and let $\varphi_{r_{0}, K_{0}}(t, x)$ be such that: (1) it is in $\bar{C}^{2}\left(D_{r_{0}, K_{0}}-\{0\}\right)$, (2) its carrier is contained in $D_{r_{0}, K_{0}}$, (3) $\varphi_{r_{0}, K_{0}} \equiv 1$ in $D_{\frac{1}{2} r_{0}, 2 K_{0}}-\{0\}$ and (4) $v=u \Psi_{r_{0}, K_{0}}$ also satisfies the condition (1.2).

Furthermore let $f$ be a monotone decreasing, smooth function such that

$$
f(t)=1 \quad \text { for } t \leqq \frac{2}{3}, \quad f(t)>0 \text { for } t<1 \text { and } f(1)=0 .
$$

Let $\alpha(t)=\alpha f(t)+(n-2)$. Finally let $\varphi(t)$ be a monotone increasing, smooth function such that

$$
\varphi(t)=t \quad \text { for } \quad t \leqq \frac{1}{4}, \quad \varphi(t)=1 \quad \text { for } \quad t \geqq \frac{1}{2}
$$

and let $\Phi_{\alpha}(t)=\varphi(t)^{2 \alpha} e^{k t}$. Then we see the following

Lemma 3. For sufficiently small $r_{0}$ and sufficiently large $K_{0}$ and $k$ there is a constant $\alpha_{0}$ such that for any $\alpha>\alpha_{0}$,

$$
\begin{aligned}
& \alpha^{2} k K_{1} \iint_{D r_{0}, K_{0}}|v|^{2} r^{-\alpha(t)} \Phi_{\alpha}(t) d x d t \\
\leqq & \left.\iint_{D r_{0}, K_{0}}|| L_{1}(v)\right|^{2} r^{2-\alpha(t)} \Phi_{\alpha}(t) d x d t+\left.\alpha^{2} K_{2} \int_{D r_{0}}|v|^{2} r^{-\alpha(t)} \Phi_{\alpha}(t) d x\right|_{t=1},
\end{aligned}
$$


where $K_{0}, K_{1}, K_{2}$ are constant numbers depending only the derivatives with respect to $t, r$, and $\varphi_{o}$, of $q$ of order $\leqq 1$, the derivatives with respect to $t, r$ of the coefficients of $N$, and $f$ of order $\leqq 1$, which are independent of systems of polar-coordinates $\left\{\varphi_{p}\right\}$. (Here and in the following proofs we denote such constants by $K$.)

(Outline of the proof). By the usual limit processes $[1,2]$ we may assume that the coefficients of $L_{1}$ and $v$ are sufficiently smooth. Let $\beta(t)=\frac{1}{2}(\alpha(t)-n+2)$ and $u=r^{\beta(t)} z$. Then we see that

$$
\begin{aligned}
& \iint\left|L_{1}(v)\right|^{2} r^{2-\alpha(t)} \Phi_{\alpha}(t) d x d t \\
\geqq & \iiint\left\{\left|q r^{2} z_{\mid t}\right|^{2}+\left|L^{* *} z\right|^{2}+2 L^{*} z \cdot L^{* *} z-2 r^{2} z_{\mid t} \cdot q \cdot\left(L^{*} z+L^{* *} z\right)\right\} \cdot \\
& \cdot r^{-1} \Phi_{\alpha}(t) d O_{1} d r d t,
\end{aligned}
$$

where

$$
\begin{aligned}
& L^{*} z=r\left(r z_{\mid r}\right)_{\mid r}+N z+\left[\frac{\alpha^{2}-(n-2)^{2}}{4}-q \alpha \frac{f^{\prime}(t)}{2} r^{2} \log r\right] z, \\
& L^{* *} z=\alpha r z_{\mid r} .
\end{aligned}
$$

From $\varphi^{\prime} \geqq 0$ it implies that for any $K$ there is a number $k_{0}$ such that for $k>k_{0}$

$$
\left(q \Phi_{\alpha}\right)_{\mid t}-K\left(q \Phi_{\alpha}\right) \geqq \frac{1}{2}\left(q \Phi_{\alpha}\right)_{\mid t} .
$$

Therefore by partial integrations with respect to $t$ and $r$ and from III in $\S 2$ and the relation $f^{\prime} \leqq 0$, it follows that

$$
\begin{aligned}
& \quad \geqq \iiint\left\{r^{3} q^{2} \Phi_{\alpha}\left(z_{\mid t}\right)^{2}+\alpha^{2} r \Phi_{\alpha}\left(z_{\mid r}\right)^{2}-2(\alpha-2) r^{2} q \Phi_{\alpha} z_{\mid r} \cdot z_{\mid t}\right. \\
& \left.\quad+2 r^{3}\left(q \Phi_{\alpha}\right)_{\mid r} z_{\mid r} \cdot z_{\mid t}-r^{3}\left(q \Phi_{\alpha}\right)_{\mid t}\left(z_{\mid r}\right)^{2}-\alpha K_{3} r^{2}\left(q \Phi_{\alpha}\right)_{\mid t} z^{2}\right\} d O_{1} d r d t \\
& \quad-\alpha^{2} K_{4} \iiint r \Phi_{\alpha} \cdot z^{2} d O_{1} d r d t \\
& +\iiint\left\{-\alpha m_{0} \Phi_{\alpha} \cdot z \cdot M z+r\left(q \Phi_{\alpha}\right)_{\mid} z \cdot M z+r q \Phi_{\alpha} z \cdot M_{\mid t} z\right\} d O_{1} d r d t \\
& +\iint\left\{q r \Phi_{\alpha} \cdot z \cdot r\left(r z_{\mid r}\right)_{\mid r}+2 r\left(q \Phi_{\alpha}\right) z^{2}-r^{3}\left(q \Phi_{\alpha}\right)_{\mid r} z \cdot z_{\mid r}\right. \\
& +\left(q \Phi_{\alpha}\right)_{\mid r} r^{2} z^{2}-r q \Phi_{\alpha} \cdot z \cdot M z+r q \Phi_{\alpha} \cdot \frac{\alpha^{2}-(n-2)^{2}}{4} \cdot z^{2} \\
& \left.-\alpha r^{2} q \Phi_{\alpha} \cdot K_{5} z^{2}\right\}\left.d O_{1} d r\right|_{t=1} .
\end{aligned}
$$

Furthermore we note that for sufficiently small $r_{0}$, for sufficiently large $K_{0}$ and $k$, there is a number $\alpha_{0}$ such that for any $\alpha>\alpha_{0}$

$$
\begin{gathered}
\alpha \Phi_{\alpha}-\left|r^{2}\left(q \Phi_{\alpha}\right)_{\mid t}\right| \geqq 0, \\
m_{0} \alpha \Phi_{\alpha}-\left|r\left(q \Phi_{\alpha}\right)_{\mid t}\right|-r q \Phi_{\alpha} K \geqq 0 .
\end{gathered}
$$

From $(3.4)_{1},(3.4)_{2}$ and II in $\S 2$, it follows that

$$
\geqq K_{6} \alpha^{2} k \iiint r z^{2} \Phi_{\alpha} d O_{1} d r d t-\left.K_{7} \alpha^{2} \iint r z^{2} \Phi_{\alpha} d O_{1} d r\right|_{t=1},
$$


which implies (3.1).

4. The second inequality. Let $r_{0}$ and $K_{0}$ be fixed numbers such that for sufficiently large $k$ and $\alpha_{0},(3.2)$ is valid.

Then using the relation: $f(1)=0, f(t)>0$ for $t<1$ and $\varphi(t)=1$ for $t \geqq \frac{1}{2}$,

we see that even if $\left.\int|v|^{2} d x\right|_{t=1} \neq 0$, there is an interval $[c, d]$ $\left(\frac{1}{2}<c<d<1\right)$ such that for any $k$ and for any $\alpha\left(>\alpha_{0}(k, u)\right)$

$$
\left.\int|v|{ }^{2} r^{-\alpha(t)} \Phi_{\alpha}(t) d x\right|_{t=1} \leqq\left.\int|v|^{2} r^{-\alpha(t)} \Phi_{\alpha}(t) d x\right|_{t}(t \in[c, d]) .
$$

Therefore from (3.2) it follows that for sufficiently large $k$ there is a constant $K_{8}$ and $\alpha_{0}$ such that for $\alpha>\alpha_{0}$

$$
\alpha^{2} K_{8} k \iint|v|^{2} r^{-\alpha(t)} \Phi_{\alpha}(t) d x d t \leqq \iint\left|L_{1}(v)\right|^{2} r^{2-\alpha(t)} \Phi_{\alpha}(t) d x d t
$$

Then from (3.1), (4.1) and (3.4) $)_{1}$ we see the following

Lemma 4. For sufficiently small $r_{0}$ and for sufficiently large $K_{0}$ and $k>k_{0}$, there are constants $K_{9}$ and $\alpha_{0}$ such that for $\alpha>\alpha_{0}$

$$
\begin{aligned}
& \iint\left(\frac{\alpha^{2}}{r_{0}^{2}}|v|^{2}+\left|v_{\mid x_{i}}\right|^{2}\right) r^{2-\alpha(t)} \Phi_{\alpha}(t) d x d t \\
\leqq & k^{-\frac{1}{2}} K_{9} \iint\left|L_{1}(v)\right|^{2} r^{2-\alpha(t)} \Phi_{\alpha}(t) d x d t,
\end{aligned}
$$

where $k_{0}$ depends on $u$ and $K$.

5. The proof of Theorem. In this section we use the notations in $\$ 1$ and $\S 2$. By (1.1) we may assume that for some $r_{0}, \varepsilon$

$$
\left|L_{1}(u)\right| \leqq M\left\{|u|+\sum_{i}^{n}\left|\frac{\partial u}{\partial y_{i}}\right|\right\} \quad \text { for } t \in[-\varepsilon, 1+\varepsilon] \text { and } r \leqq r_{0}
$$

where $2 K_{0} r_{0}<\frac{1}{4}$.

Then from Lemma 4 we see that for any $\alpha\left(>\alpha_{0}\left(K, r_{0}, k\right)\right)$

$$
\begin{gathered}
\iint_{D_{r_{0} / 2,2 K_{0}}}\left(|u|^{2}+\left|u_{\mid y_{i}}\right|^{2}\right) r^{2-\alpha(t)} \Phi_{\alpha} d y d t \\
\leqq k^{-\frac{1}{2}} K \iint_{D_{r_{0}, K_{0}}}\left|L_{1}(v)\right|^{2} r^{2-\alpha(t)} \Phi_{\alpha} d y d t \\
\leqq k^{-\frac{1}{2}} K \iint_{D_{r_{0}, K_{0}}-D_{r_{0} / 2,2 K_{0}}}\left|L_{1}(v)\right|^{2} r^{2-\alpha(t)} \Phi_{\alpha} d y d t \\
\quad+k^{-\frac{1}{2}} K \cdot M \iint_{D r_{0} / 2,2 K_{0}}\left(|u|^{2}+\left|u_{\mid y_{i}}\right|^{2}\right) r^{2-\alpha(t)} \Phi_{\alpha} d y d t
\end{gathered}
$$

Accordingly choosing $k$ sufficiently large such that

it follows that for any $\alpha>\alpha_{0}$

$$
2 K \cdot M<k^{\frac{1}{2}},
$$




$$
\begin{aligned}
& \frac{1}{2}\left(\frac{r_{0}}{3 K_{0}}\right)^{2-\alpha-(n-2)} \int_{\frac{1}{4} \leqq t \leq \frac{2}{3}, r \leqq r_{0} / 3 K_{0}}\left\{|u|^{2}+\left|u_{\mid y_{i}}\right|^{2}\right\} e^{k t} d y d t
\end{aligned}
$$

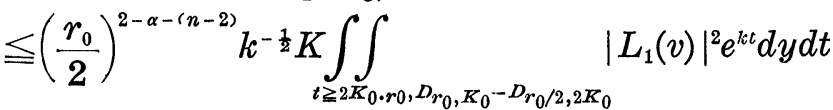

$$
\begin{aligned}
& +\left(\frac{1}{2 K_{0}}\right)^{-\alpha-n} k^{-\frac{1}{2}} K \iint_{t \leq 2 K_{0} \cdot r_{0}, D_{r_{0}}, K_{0}-D_{r_{0} / 2,2 K_{0}}}\left|L_{1}(v)\right|^{2} e^{k t} d y d t .
\end{aligned}
$$

Therefore tending $\alpha \rightarrow \infty$, we see that

$$
u(t, y)=0 \text { for } t \in\left[\frac{1}{4}, \frac{2}{3}\right], \quad r \leqq r_{0} / 3 K_{0} .
$$

Since, in the above proof, the numbers $\left\{\varepsilon, \frac{1}{4}\right\}$ and $\frac{2}{3}$ may be replaced by arbitrary small and large numbers respectively, we see that $u(t, x)=0$ in a neighbourhood of $C$ in $(a, b) \times R_{x}$. Then by a topological argument and from Lemma 1 and Lemma 4 also, we see that $u(t, x) \equiv 0$ in the horizontal component stated in $\$ 1$.

Another detailed proof of Theorem and the results in my previous paper [4] with other consequences will be published in the Osaka Mathematical Journal next year.

\section{References}

[1] N. Aronszajn: A unique continuation theorem for solutions of elliptic partial differential equations or inequalities of second order, Tech. Report 16, Univ. Kansas (1956).

[2] H. O. Cordes: Über die eindeutige Bestimmtheit der Lösungen elliptischer Differentialgleichungen durch Anfangsvorgaben, Nachr. Akad. Wiss. Göttingen, Math-phys., KI, IIa, no. 11, 239-258 (1956).

[3] E. Heinz: Ưber die Eindeutigkeit beim Cauchysehen Anfangswert-problem einer elliptischen, Differentialgleichung zweiter Ordnung, Nachr. Akad. Wiss. Göttingen, no. 1, 1-12 (1955).

[4] T. Shirota: A remark on the abstract analyticity in time for solutions of a parabolic equation, Proc. Japan Acad., 35, no. 7, 367-369 (1959). 